\title{
La sacralisation du territoire jordanien
}

Reconstruction des lieux saints nationaux, 1980-2006

\section{Norig Neveu}

\section{(2) OpenEdition}

Journals

Édition électronique

URL : http://journals.openedition.org/assr/22357

DOI : $10.4000 /$ assr.22357

ISSN : $1777-5825$

Éditeur

Éditions de l'EHESS

Édition imprimée

Date de publication : 1 septembre 2010

Pagination : 107-128

ISBN : 978-2-7132-2255-9

ISSN : 0335-5985

Référence électronique

Norig Neveu, "La sacralisation du territoire jordanien », Archives de sciences sociales des religions [En ligne], 151 | juillet-septembre 2010, mis en ligne le 20 octobre 2010, consulté le 30 avril 2019. URL : http://journals.openedition.org/assr/22357 ; DOI : 10.4000/assr.22357 


\section{Norig Neveu}

\section{La sacralisation du territoire jordanien Reconstruction des lieux saints nationaux, 1980-2006}

La topographie musulmane du territoire jordanien connaît des changements notables depuis le début des années quatre-vingt-dix. Le ministère des waqf-s ${ }^{1}$ a rénové ou reconstruit une quarantaine de mausolées de prophètes antéislamiques ou de Compagnons du Prophète, sous l'égide du prince Ghazi b. Muhammad ${ }^{2}$, responsable des affaires religieuses à la couronne et directeur du Comité Royal pour la construction des mosquées et des mausolées de prophètes, Compagnons et martyrs ${ }^{3}$. Ces mausolées sont situés pour la plupart dans la vallée du Jourdain, le long de la frontière avec Israël, et attestent de l'intérêt politique de la monarchie hachémite pour les lieux saints nationaux. Un même regain d'intérêt pour la question des lieux saints est observé dans l'ensemble du Moyen-Orient. En Palestine, les mausolées de nabi Salih et de nabi Musa ont fait l'objet d'un jeu politique de la part de l'Autorité palestinienne afin d'exacerber le sentiment national (Aubin-Boltanski, 2004). En Syrie, de nombreuses rénovations ont été effectuées pour accueillir des pèlerins chiites (Ababsa, 2001 ; Mervin, 1996 ; Pinto, 2007). Cependant, si la fondation et la reconstruction de lieux de culte sont un phénomène répandu au Moyen-Orient, elles sont particulièrement massives en Jordanie et se trouvent au centre d'enjeux politiques et économiques spécifiques.

Dans son discours officiel, la monarchie souligne fortement la relation historique de la famille hachémite aux lieux saints et l'appartenance à l'A $\mathrm{bl}$ al-bayt ${ }^{4}$,

1. Waqf (plur. awqāf) : dans la loi islamique, bien de «main morte » fondé pour une œuvre religieuse (œuvre de charité, service public, etc.) La nature et la gestion des waqf-s sont très variées. Aujourd'hui, le ministère des waqf-s gère ces œuvres et leurs revenus.

2. Petit-fils du roi Talal, il est, depuis 2003, conseiller et représentant du roi Abdallah II.

3. Nous le désignerons par Comité royal. Ses membres l'appellent également Comité de rénovation alors que les travaux consistent plutôt en une reconstruction du mausolée original. Nous parlerons ainsi de reconstruction pour évoquer les travaux de ce comité.

4. Les Hachémites sont des sharif-s, ce qui signifie qu'ils appartiennent à la descendance du Prophète Muhammad. Selon les traditions, ils sont présentés comme les descendants de la fille du Prophète, Fatima, ou comme les descendants de Hashim b. Abd Manaf, l'arrière-grandpère de Muhammad, héraut de l'antéislam. Au moment de la première prédication de l'islam, les Banu Hashim appartenaient à la tribu de Qoreïch, qui était alors la plus importante du Hedjaz (Sourdel, 2004). 
la lignée des descendants du Prophète, ce qui lui sert d'argument d'autorité pour se prononcer non seulement sur des questions politiques mais aussi religieuses ${ }^{5}$. Pendant environ dix siècles et jusqu'en 1924, la famille hachémite a détenu la charge de chérif de la Mecque et Médine, les haramain de l'islam (Dawn, 1971). Cette charge lui conférait un rôle religieux important, notamment par la gestion du pèlerinage, le hajj, et l'entretien des lieux saints. Après la bipartition de la Palestine, entre 1948 et 1967, les lieux saints de Jérusalem-Est sont tombés sous autorité jordanienne. En se présentant comme garante héréditaire des lieux saints (Katz, 2005 : 214), la monarchie peut donc revendiquer un lien historique continu ${ }^{6}$ avec ceux-ci et jouer ainsi le rôle de porte-parole de la communauté musulmane.

Dans les années cinquante, la monarchie jordanienne avait adopté une politique de rénovation et de construction des lieux saints nationaux chrétiens afin de promouvoir le tourisme religieux. Après l'annexion de la Cisjordanie par le royaume hachémite, le roi Abdallah $\mathrm{I}^{\mathrm{er}}$, puis le roi Hussein ont utilisé Jérusalem comme capitale spirituelle du royaume. Les lieux saints, chrétiens comme musulmans, font l'objet de nombreux projets de rénovation. Entre 1948 et 1967, la monarchie encourage le tourisme vers la ville sainte et revendique l'assimilation de son pays à la Terre sainte chrétienne. Ce dessein sera, pour partie, consacré par le pèlerinage du pape Paul VI en Terre sainte (incluant la Jordanie), en 1964, et plus tard par ceux de Jean-Paul II (2000) et Benoît XVI (2009). Après 1967, les découvertes archéologiques au site du baptême du Christ ${ }^{7}$ et au Mont Nébo entraînent une redéfinition de cette notion de Terre sainte jordanienne, recentrée sur le territoire national dans ses nouvelles frontières. La sacralisation musulmane du territoire suit des dynamiques similaires. Nous verrons qu'elle est cependant porteuse d'une idéologie particulière et contribue à la réécriture de l'histoire religieuse jordanienne.

On adoptera une approche historique pour tenter de comprendre comment la création d'une topologie sacrée musulmane s'accompagne d'un discours nationaliste centré sur la monarchie. Les données empiriques sont constituées à partir de l'analyse de textes (la littérature produite par le ministère des waqf-s et par le ministère du Tourisme et des Antiquités concernant les lieux saints jordaniens, ainsi qu'une série de documents fournis par le Comité royal). Nous avons également effectué, entre 2006 et 2009 , de nombreux entretiens auprès des ingénieurs

5. Rappelons ici que la légitimité religieuse des Hachémites est remise en cause par les islamistes jordaniens.

6. Ce lien historique avec la ville de Jérusalem a d'ailleurs été renforcé après l'enterrement du Shérif Hussein de la Mecque à Jérusalem, en 1931, et l'assassinat du roi Abdallah I ${ }^{\text {er }}$, en 1951, devant la mosquée al-Aqsa. La perte des lieux saints de Jérusalem, en 1967, fut considérée comme une importante défaite pour les Hachémites.

7. C'est à l'initiative du prince Ghazi b. Muhammad et du père Piccirillo, archéologue franciscain, que fut créé un parc national autour de ce site, considéré aujourd'hui comme le véritable site du baptême et légitimé par Benoît XVI en mai 2009. 
du Comité royal, des fonctionnaires du ministère des waqf-s, des architectes en charge des travaux mais aussi auprès des professionnels du tourisme. Nous avons, par ailleurs, mené des observations directes sur l'usage de ces sites par les populations locales et les touristes.

Cet article se propose de décomposer le mouvement de sacralisation du territoire jordanien et d'en cerner les différents enjeux. On tentera d'analyser son influence sur la réécriture de l'histoire jordanienne et sur la redéfinition de l'usage de ces sites. Après avoir décrit les modifications de la topographie musulmane à travers l'examen des nouvelles pratiques artistiques et architecturales, on tentera de déterminer les effets de l'établissement du territoire jordanien comme Terre sainte musulmane sur la réécriture de l'histoire islamique du royaume. Comment ces sites permettent-ils à la monarchie de créer une continuité au niveau de l'histoire islamique nationale à différentes échelles ? Comment la reconstruction des mausolées peut-elle représenter un cas de désappropriation de lieux de piété locaux afin d'y développer le tourisme religieux ? Quel est l'impact géostratégique de cette nouvelle forme de tourisme?

\section{Reconstruire, investir, redéfinir}

\section{Islamisation physique du territoire jordanien}

La géographie urbaine jordanienne est marquée par un profond mouvement d'islamisation dont la ville d'Amman représente un bon exemple (Rogan, 1986). Dans les années soixante-dix, le roi Hussein décide de donner à la ville un caractère islamique afin de la rendre plus représentative des héritages culturels et religieux de ses habitants. Ce projet se concrétise par l'édification de nombreuses mosquées, entre 1980 et 1984, notamment celle du roi Abdallah I ${ }^{\text {er }}$, achevée en 1989. Les bureaux du Comité royal se trouvent dans l'enceinte même de ce bâtiment.

Rappelons ici que le projet hachémite de reconstruction des lieux saints ne concerne qu'une partie des lieux de piété nationaux. Il existe, à l'échelle locale, une multitude de lieux de culte populaire n'ayant pas été pris en compte par le ministère des waqf-s. Dans le nord du pays, où sont implantées des confréries soufies, on trouve de nombreux mausolées de saints. Les travaux de Bethany Walker (2007) sur la région de Malka au XIX ${ }^{\mathrm{e}}$ siècle ont démontré leur importance dans le dynamisme d'une ville de petite taille. La présence du mausolée du cheikh $\mathrm{Omar}^{8}$ y a notamment engendré le développement des infrastructures

8. Selon Bethany Walker (2007), le sheikh Omar est considéré comme l'un des descendants de Abd al-Qadir al-Jilani, fondateur de la confrérie soufie Qadiriyya à Baghdad au XII siècle. Le sheikh Omar dirigea l'une des branches de la confrérie dans la région de Malka dans les années 1880. Il serait mort au début du XX $\mathrm{X}^{\mathrm{e}}$ siècle. 
médicales et scolaires. À l'échelle locale, la famille se réclamant de la descendance $\mathrm{du}$ saint jouit toujours d'une grande aura. Les confréries représentent encore aujourd'hui des espaces de sociabilité privilégiés. Par ailleurs, de nombreux sites, liés à l'histoire tribale ou à une topographie sacrée locale, sont aujourd'hui laissés à l'abandon et menacés de disparition. La carte des lieux saints dressée à partir de la nouvelle topographie religieuse officielle (fig. 1) n'est donc pas exhaustive. Elle n'intègre pas non plus des lieux de pèlerinage autrefois importants à l'échelle régionale. Le pèlerinage au mont Harun, par exemple, qui se déroulait deux fois par an et regroupait l'ensemble des tribus de Wadi Musa, Pétra et al-Shubak, dans le sud jordanien, a pris fin. Mais, comme le démontre Anna Ohannessian (1995), certains de ces lieux ont pu être réinvestis par les communautés locales. L'auteure décrit les diverses fonctions des lieux saints pour la tribu des Balawneh.

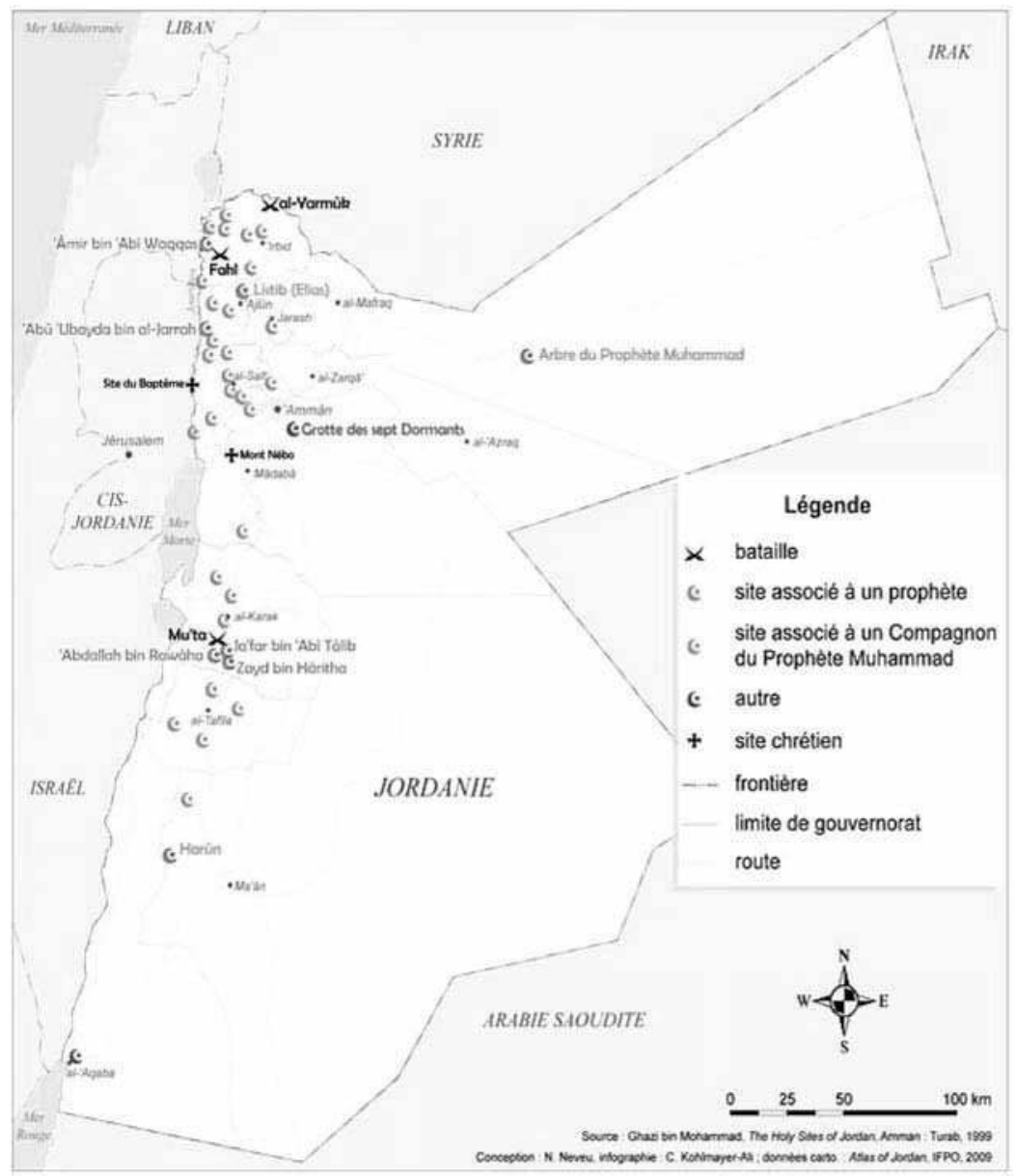

Figure 1. Carte de la topologie musulmane officielle 
Le mausolée du cheikh Falali al-Faqir, un $w \bar{a} l \bar{\imath}^{9}$, leur servait de grenier, situé à proximité des mausolées d'Abu 'Ubayda b. al-Jarrah où se trouvait le cimetière de la tribu. On comprend ainsi l'importance des charges symboliques et économiques du mausolée au niveau local et régional. Le réinvestissement et l'officialisation de certains types de lieux saints seulement reflètent la volonté de la monarchie de laisser de côté des lieux et des rituels religieux locaux.

\section{Reconstruction officielle et topologie}

À partir des années quatre-vingt, le roi Hussein commence à promouvoir la reconstruction des mausolées de prophètes et de Compagnons du Prophète. Selon un article du journal al-Ghad, daté du 22 avril 2005 :

«En 1984, sa majesté le roi Hussein a adressé un communiqué au Premier ministre, dans lequel il lui ordonna de former un comité chargé de la reconstruction des mausolées des Compagnons du Prophète et des martyrs qui conquirent le territoire jordanien. Il offrit ses revenus personnels dans une action de charité pour reconstruire la mosquée et la tombe de Ja'far b. Abi Talib à al-Karak » (al-'Adwa, al-Zanibat, 2005, trad. de l'auteure).

En 1992, le monarque envoie un second communiqué au Premier ministre pour insister sur l'importance de ces rénovations :

" ... sur le sol vertueux de Jordanie afin que ces tombes deviennent des marqueurs de la droiture et des témoins de la vérité, élevées à la gloire des dignes prophètes et Compagnons ; reliant petits-fils et grands-pères » (ibid.)

Le Comité royal, formé en 1994, est constitué d'une dizaine de personnes sous la direction des princes Ra'ed b. Hussein et Ghazi b. Muhammad. Il dépend du ministère des waqf-s et regroupe des membres de ce ministère, du ministère du Tourisme et des Antiquités, du ministère des Travaux publics, ainsi que des ingénieurs. La majorité des architectes jordaniens de renom, dont Rasem Badran et Ayman Zuayter, sont sollicités pour dessiner les plans des nouveaux complexes religieux. Après avoir lancé un appel à projet, le comité choisit les architectes en charge des rénovations. Ce sont alors les membres du comité exécutif, constitué d'ingénieurs, dont Ghasan al-Burani et Adli Abu Shadi, qui dirigent les travaux. Le projet de reconstruction de ces mausolées est réalisé en trois étapes et débute, en 1996, avec leur rénovation et la création de jardins adjacents. La première étape concerne les mausolées de trois commandants morts à la bataille de Mu'ta : Ja'far b. Abi Talib, Abdallah b. al-Rawaha, Zayd b. al-Haritha ${ }^{10}$, ainsi que celui d'Abu 'Ubayda b. al-Jarrah, commandant militaire des batailles de Fahl et de

9. Wâlī : littéralement «ami de Dieu », celui qui est proche de lui ; traduit par «saint » en français.

10. La bataille de Mu'ta se déroula en 629, du vivant du Prophète : ce fut une défaite pour les troupes musulmanes qui, se trouvant en minorité face aux troupes byzantines, durent battre en retraite. 
Yarmouk ${ }^{11}$. Ces deux sites religieux sont centraux dans le processus de création d'une topographie sainte en Jordanie.

En 1999, au début du règne du roi Abdallah II, un deuxième projet regroupe dans une même enceinte deux des mausolées (ceux de Ja'far b. Abi Talib et de Zayd b. al-Haritha) et une mosquée. De nombreuses commodités destinées aux futurs visiteurs y sont aménagées : cafétérias, boutiques, bibliothèques, etc. Ce projet nécessite de déplacer la population de la zone. La troisième étape, prévue pour 2010, devrait réunir les trois mausolées, des jardins permettant la circulation d'un édifice à l'autre. De dimensions modestes à l'origine, ils formeront un vaste ensemble religieux avec d'importantes capacités d'accueil.

Le mausolée d'Abu 'Ubayda b. al-Jarrah est développé sur un schéma à peu près similaire, une école coranique et une bibliothèque ayant été accolées au monument préexistant, entouré d'un mur d'enceinte. L'ensemble des autres sites rénovés, de dimensions plus modestes, répondent à un même plan organisationnel bien que les architectes qui les ont conçus soient différents. À côté de chaque mausolée s'élève une mosquée pouvant accueillir en moyenne trois cents fidèles ; certains sont dotés de salles de prière pour les femmes. Ces complexes religieux comprennent aussi des salles de repos, des jardins et, dans la plupart des cas, des commerces et des cafétérias mais qui n'ont pas encore été mis en fonction.

\section{Vers la définition d'une architecture islamique jordanienne?}

De nombreux architectes jordaniens ont participé à la création de cette nouvelle topologie sacrée. Ces constructions ont été effectuées sans prendre en compte la notion de patrimoine historique. L'architecte Rasem Badran, qui a réalisé les plans du site d'Abu 'Ubayda b. al-Jarrah et de celui dédié aux commandants de la bataille de Mu'ta, explique : "les mausolées anciens étaient de l'architecture locale, sans prétention, nous avons décidé de redonner de la noblesse et de l'importance au bâtiment pour honorer la personne illustre qui y est enterrée ${ }^{12}$.

Dans la majorité des cas, la reconstruction du site a provoqué la destruction du bâtiment original. L'exemple du mausolée d'Amir b. Abi Waqqas atteste de la métamorphose de l'architecture du bâtiment. La coupole, qubba, que l'on peut voir sur la photographie de 1972 (fig. 2) n'existe plus après les travaux (fig. 3). On note de nombreux cas similaires de disparition des formes anciennes de qubba (à l'exception du mausolée du nabi Harun). Ces qubba-s ont été remplacées par des coupoles vertes, qui correspondent, selon Ghasan al-Burani ${ }^{13}$,

11. La bataille de Fahl (635) contre les troupes byzantines permit aux troupes musulmanes d'avancer la conquête du Bilad al-Sham. La bataille de Yarmouk (636) permit d'asseoir une présence musulmane définitive dans la région.

12. Entretien avec Rasem Badran, architecte, Dar al-Omran, Amman, 14 mai 2009.

13. Entretien avec Ghasan al-Burani, ingénieur du Comité royal technique, Amman, juillet 2006. 


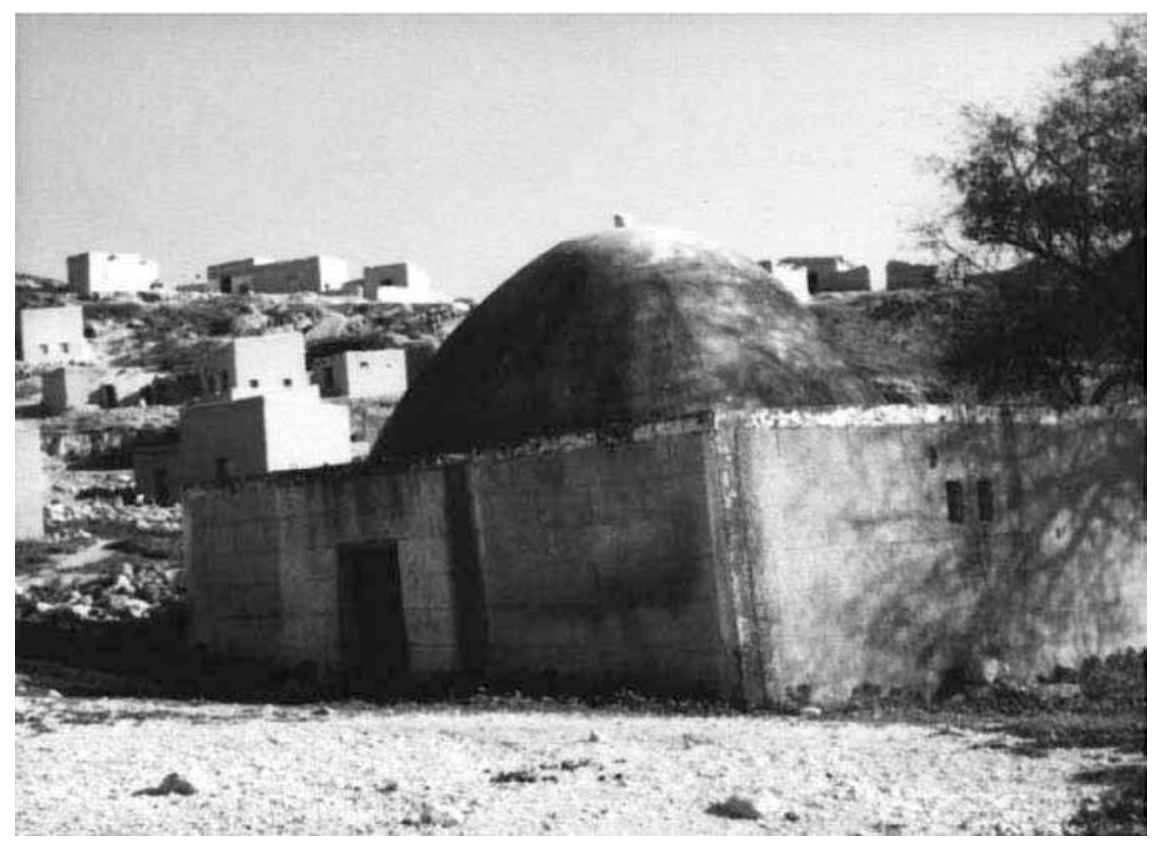

Figure 2. Mausolée d'Amir b. Abi Waqqas en 1972 (Photographie du Comité royal)

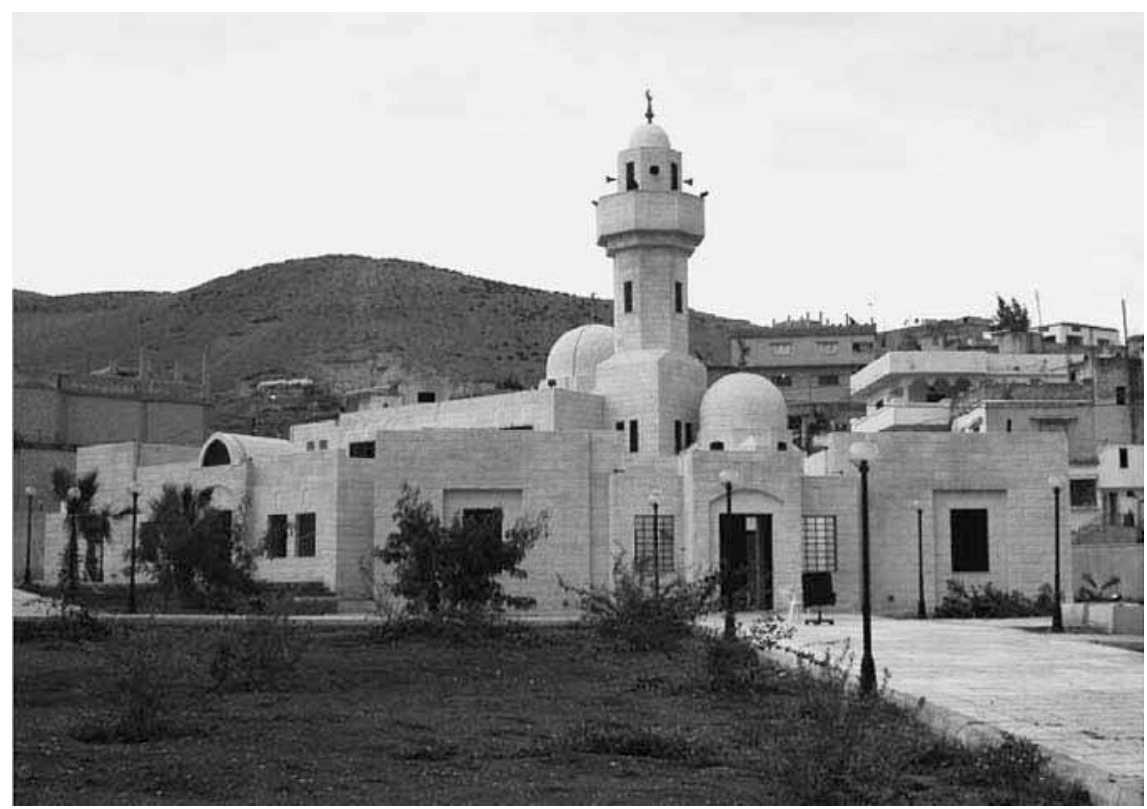

Figure 3. Mausolée d'Amir b. Abi Waqqas en 2004 (Photographie du Comité royal) 
à la norme islamique contemporaine. Cette entreprise de reconstruction est caractéristique de la tentative d'harmonisation de l'architecture religieuse jordanienne et de la mise en œuvre de modèles et normes officiels.

Dans le cas des mausolées dédiés aux commandants de la bataille de Mu'ta et à Abu 'Ubayda b. al-Jarrah, on retrouve, après la reconstruction, quelques éléments de l'architecture préexistante. Grâce à l'étude des photographies du mausolée de Ja'far b. Abi Talib, on peut constater que le bâtiment préexistant à double coupole, datant probablement du début du $\mathrm{XX}^{\mathrm{e}}$ siècle (fig. 4 et 5), a été conservé. Cependant, l'adjonction d'un complexe de grandes dimensions autour de ces sépultures en modifie profondément le style. Les formes architecturales originelles ont ainsi été intégrées dans un ensemble contemporain qui en efface les particularités.

Dans un article consacré aux mosquées ibadites ${ }^{14}$, Virginie Prévost (2009) souligne la menace de disparition de ces édifices, aujourd'hui, en Algérie, en Libye et en Tunisie, soit par abandon, soit en raison de destructions suivies de reconstructions selon un modèle nord-africain classique. L'auteure définit les attributs de l'art ibadite comme représentatifs de la particularité des conceptions religieuses de la société bédouine ibadite. Le cas jordanien relève de dynamiques similaires, bien qu'ici la redéfinition sociale souhaitée par la monarchie soit véhiculée par la nouvelle tradition architecturale : c'est l'art officiel qui tend à établir la culture et les traditions de la communauté. Il s'agit d'harmoniser l'architecture religieuse sur l'ensemble du territoire jordanien en effaçant les particularités régionales. La reconstruction des mausolées jordaniens représente un cas de désappropriation par l'État de lieux de piété populaire à des fins touristiques. Les caractéristiques locales sont effacées pour que ces sites apparaissent comme des éléments centraux du patrimoine islamique.

Pour ces reconstructions, le Comité royal a reçu l'aide de l'Institute of Islamic Tradition Art. Créé, en 1997, à l'initiative du prince Ghazi b. Muhammad, le but de ce centre de formation est, selon Farid Medea ${ }^{15}$, membre et professeur de l'Institut, de donner une continuité à la tradition artistique. L'établissement s'est notamment occupé de la reconstruction à l'identique du minbar ${ }^{16}$ de la mosquée d'al-Aqsa envoyé à Jérusalem en 2007. Il s'est aussi chargé de la construction des minbar-s de nombre de sites rénovés en tentant d'utiliser des formes géométriques classiques, tout en jouant sur l'utilisation innovante de couleurs originales dans le travail du bois et de la pierre, et en mélangeant ces matériaux. La dynastie souhaite, par le biais de cet institut et du Comité royal,

14. Al-Ibâdiyya (les ibadites) est une secte de l'islam présente principalement en Afrique orientale, en Oman, en Tripolitaine et en Algérie du sud. Les ibadites sont l'une des branches des kharijites (T. Lewicki, 1971).

15. Entretien avec Farid Medea, membre de l'Institute of Islamic Tradition Arts et professeur, Amman, 28 avril 2006.

16. Chaire à partir de laquelle l'imam prononce son prêche. 


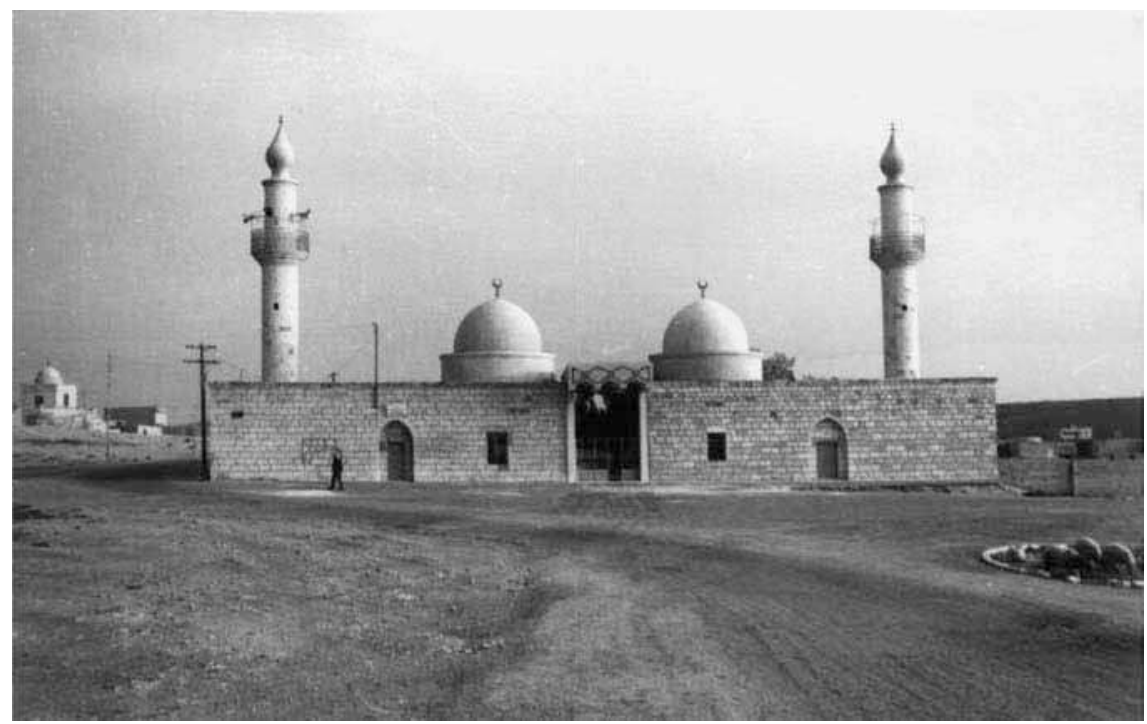

Figure 4. Mausolée de Ja'far b. Abi Talib dans les années soixante (Photographie du Comité royal)

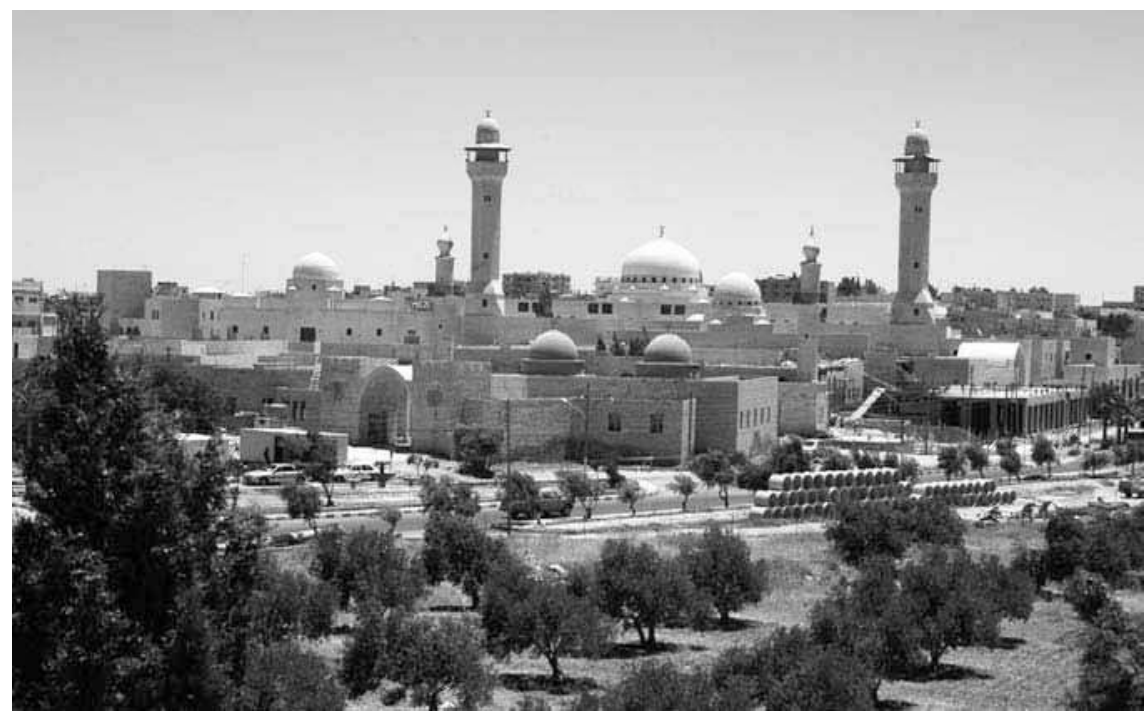

Figure 5. Mausolées dédiés aux commandants de la bataille de Mu’ta, en 2001 (Photographie du Comité royal)

introduire de nouvelles formes d'art et d'architecture islamiques. Celles-ci se traduiraient par la modernisation de certaines traditions artistiques. Le développement d'un style architectural propre à la monarchie est lié à l'affirmation de l'identité politique et culturelle hachémite. Il se veut représentatif des conceptions 
de l'islam officiel exprimées dans le message d'Amman ${ }^{17}$, fer de lance de la monarchie. On peut ainsi penser que ces reconstructions sont également un moyen pour la monarchie de contrôler les pratiques religieuses des populations locales.

\section{Mausolées, Terre sainte et construction nationale}

\section{La Jordanie : Terre sainte musulmane}

Dans un guide émis par le ministère des waqf-s, on peut lire :

«La Jordanie : cette terre bénie, cette continuité historique, ce patrimoine civilisationnel et cette mémoire accumulée de grands événements de l'histoire de l'Humanité... Oui... c'est le cœur de la région qui a été honoré par la révélation de Dieu ToutPuissant à nombre de ses prophètes et messagers... C'est la porte de la conquête islamique, l'un des axes les plus importants de l'histoire musulmane, suivant la révélation par Dieu du message islamique » (ministère des waqf-s, $2000:$ 7) (trad. de l'auteure).

Cette citation résume avec clarté l'objectif hachémite : le territoire jordanien est présenté comme sanctifié par la présence de mausolées et de sites significatifs pour l'histoire islamique. Par là, le lien est fait entre l'histoire de la Jordanie contemporaine et celle des premiers temps de l'islam. Dans la littérature concernant la rénovation des lieux saints, le récit du martyre des Compagnons du Prophète aux batailles de Fahl, de Yarmouk et de Mu'ta tient une place importante. Cette insistance vise à définir la Jordanie comme un pays central de l'histoire et de la conquête islamiques du Bilad al-Sham:

"Il est situé au centre du monde arabe et islamique, sur la route menant au hajj et à l'umra vers la Mecque; le pont tendu entre les deux qibla-s, Jérusalem, qui est le troisième sanctuaire après ceux de La Mecque et Médine, et la sainte mosquée, qui fut la première maison érigée par Dieu sur terre. C'est aussi la route suivie par le Prophète arabe hachémite dans son voyage vers Jérusalem. Sur ce territoire, le grand Prophète voyagea deux fois, vers Busra al-Sham, avant sa mission religieuse (...)» (ibid.)

Cette définition présente la Jordanie comme appartenant à une unité territoriale qui désigne ici la Terre sainte musulmane (al-ard al-muqaddassa) : entre les lieux saints du Hedjaz et ceux de Jérusalem. On peut comprendre, en ce sens, l'attachement de la dynastie à la ville de Jérusalem. L'expression al-ard al-muqaddassa apparaît dans le Coran, mais ne s'applique pas à un territoire clairement circonscrit. Il existe plusieurs traditions liées à la définition de l'arḍ al-muqaddassa (AubinBoltanski, 2004 : 32). Une première version la présente comme un large territoire

17. Le message d'Amman a été prononcé, en novembre 2004, dans la mosquée Abdallah I Ir afin de : "clarifier pour le monde moderne la vraie nature de l'islam et la nature du vrai islam » (trad. de l'auteure). Il insiste sur la nécessité du dialogue et de la tolérance entre les différents courants et écoles juridiques de l'islam. C'est dans ce message que sont exprimées les conceptions de l'islam officiel selon la monarchie. www.ammanmessage.com 
qui s'étire du nord au sud, de l'Euphrate au Hedjaz, et d'est en ouest, d'Aqaba au désert du Sinaï. Une autre version la circonscrit à la ville de Jérusalem, en s'appuyant sur la tradition du Voyage nocturne et sur le fait que Jérusalem fut la première qibla. Le processus de sacralisation du territoire s'appuie aussi sur des versets coraniques et des hadiths. L'exégèse de la sourate coranique ${ }^{18}$ relative au Voyage nocturne de Muhammad vers Jérusalem permet d'apporter un argument d'autorité concernant la sacralité du territoire jordanien, situé sur le trajet du Prophète. La situation géographique du pays est donc le premier argument utilisé par la monarchie pour intégrer son pays à la Terre sainte musulmane.

Le second argument permettant de justifier les revendications hachémites est l'attachement du Prophète lui-même à ce territoire :

«Pour les musulmans, la Jordanie est un territoire béni, marqué par de nombreuses tombes et mausolées. (...) Le Prophète mentionna Amman en la comparant avec le Paradis quand il dit : "Mon bassin (au Paradis) s'étend entre Aden et Amman al-Balqa". À deux reprises, le territoire de Jordanie joua un rôle dans la vie de Muhammad » 19 (brochure du Jordan Tourism Board, 2002 : 1, trad. de l'auteure).

Cette position émanant d'une institution du ministère du Tourisme est sans ambiguïté. Le territoire jordanien est directement mis en relation avec la personne du Prophète. Celui-ci le décrit comme le Paradis terrestre. C'est dans ce cadre que le comité a ajouté à son entreprise de reconstruction un arbre situé dans la région d'al-Azraq, à al-Safawi. Selon le ministère des waqf-s (site Internet du ministère) c'est contre cet arbre que le jeune Muhammad se serait adossé alors qu'il suivait l'une des caravanes de son oncle entre le Hedjaz et la Syrie. Il aurait alors été reconnu par le moine Bahira comme portant le sceau de la prophétie (Abel, 1960). Le Comité royal a entouré cet arbre d'un grillage et construit une loge de gardien. L'arbre a dès lors été fréquemment représenté sur les documents officiels émanant du ministère des waqf-s (brochures, site Internet, prospectus). L'histoire du Prophète apparaît ici en étroite relation avec l'histoire nationale jordanienne : c'est, en tout cas, le but de cette « histoire officielle reconstruite ».

Maurice Halbwachs (1971) décrit la création de la Terre sainte biblique comme fondée sur une "histoire romancée " des événements bibliques et sur une transformation de la mémoire et des souvenirs ayant entraîné une réappropriation de l'espace historique. Selon lui, la mémoire collective chrétienne a adapté, à chaque époque, ses souvenirs, les détails de la vie du Christ et les lieux auxquels ils se rattachent, aux exigences contemporaines du christianisme et à ses aspirations. De la même manière, en Jordanie, la création d'une topologie s'appuie sur une histoire réécrite qui s'adapte aux enjeux politiques et économiques contemporains. Cependant, si les processus sont similaires dans le cas

18. Cf. Coran V, 21.

19. Ce passage fait référence à certaines traditions concernant la vie du Prophète Muhammad selon lesquelles l'ascète Hanif Zayd b. 'Amr b. Nufayl puis le moine Bahira l'auraient reconnu comme prophète alors qu'il était encore un jeune homme. 
jordanien, la mémoire collective semble avoir mis de côté les traditions populaires liées aux lieux saints reconstruits. Par exemple, la tradition locale relative à la bataille de Mu'ta insiste sur le rôle de la tribu chrétienne des 'Azayzat, dont l'ancêtre aurait aidé les troupes musulmanes contre les Byzantins (Chatelard, 2004 : 57). Le Prophète aurait alors qualifié de 'azīz (cher), l'ancêtre de cette tribu. De la même manière, il existe une topologie sacrée locale complexe autour du mont Harun. Ces traditions ont été occultées de l'histoire officielle des sites. La «topographie légendaire » jordanienne s'appuie sur une tradition récemment élaborée et matérialisée par la reconstruction de lieux saints musulmans.

Ainsi, la définition de la Jordanie comme Terre sainte musulmane se fonde sur deux arguments majeurs : la situation géographique du pays et l'attachement du Prophète lui-même à ce territoire. Dans l'une des publications du ministère des waqf-s, le Prophète est désigné de façon significative comme " arabe hachémite » (ministère des waqf-s, op. cit. : 7), valorisant ainsi le lien historique entre le Prophète et la famille royale. Cela permet à la monarchie de se présenter comme héritière et garante du message prophétique. L'élaboration de la Terre sainte jordanienne répond donc d'une construction discursive complexe permettant de mettre en relation Terre sainte et Jordanie mais aussi le Prophète et la famille royale.

\section{La Jordanie : terre de l'Ahl al-bayt}

La représentation par la famille royale du pays comme Terre sainte musulmane s'appuie sur l'assimilation du territoire à la terre de l'Abl al-bayt. Rappelons que la majorité des mausolées de prophètes et Compagnons du Prophète présents sur le territoire jordanien se situe dans la vallée du Jourdain, le long de la frontière avec Israël. Cette situation de marqueur géographique peut représenter une volonté symbolique de la part de la monarchie de s'opposer à la définition israélienne de la Terre sainte juive. Benvenisti (2000) a montré comment Israël a identifié des lieux saints sur son territoire et les territoires occupés sur lesquels ont ensuite été développés des rituels religieux. Au cours de ce processus, de nombreux lieux saints ont été réinventés, y compris par l'appropriation de lieux de culte musulman. Les lieux saints sont, ainsi, un moyen de contrôler et délimiter le territoire national. Cette invention d'une géographie religieuse justifie la présence de l'État d'Israël sur un territoire gouverné par des dynasties arabes pendant plusieurs siècles. Ainsi, de nouvelles dénominations des périodes historiques de l'antiquité en Israël ont été utilisées par les archéologues dans le but d'enraciner le peuple juif dans la région. Cet usage politique de l'histoire et de l'archéologie israéliennes a pu servir d'exemple pour les États voisins. On peut penser que la Jordanie réutilise aujourd'hui massivement cette stratégie dans le cadre des rénovations de lieux saints musulmans afin d'affirmer ses frontières nationales et d'inscrire sur le long terme la présence des Hachémites comme dirigeants politiques et militaires. 
Dans cette construction de la topologie sacrée musulmane, les sites liés aux prophètes sont assez nombreux, mais ce sont les figures des Compagnons du Prophète qui ont été valorisées. Si les membres de l'Abl al-bayt et les Compagnons du Prophète font l'objet de cultes dans l'ensemble du monde arabe, la reconstruction des mausolées de Compagnons du Prophète en Jordanie s'accompagne de l'élaboration d'un discours officiel permettant de recréer une continuité historique entre ces derniers et la monarchie hachémite.

Le ministère des waqf-s a produit une littérature dense concernant les mausolées nationaux qui exalte essentiellement la personnalité des Compagnons et leur bravoure, laissant quelque peu de côté l'évocation des prophètes. Cela peut s'expliquer par la volonté des dirigeants jordaniens de mettre en avant une spécificité dans leur définition de la Terre sainte jordanienne, par rapport à la Palestine considérée comme «Terre des prophètes » (Aubin-Boltanski, 2004 : 32). On note une compétition croissante entre les différents pays de la région, Palestine, Liban, Syrie et Jordanie, concernant le tourisme religieux. Le territoire jordanien entre, d'ailleurs, directement en concurrence avec les territoires d'outre-Jourdain dans la revendication d'appartenance à la Terre sainte, tant d'un point de vue chrétien que musulman. Les Compagnons du Prophète étant des référents exclusivement islamiques, contrairement aux prophètes qui appartiennent également au judaïsme et au christianisme, on peut penser que la Terre sainte jordanienne est élaborée discursivement autour de la spécificité purement islamique de sa topologie. Elle se distingue ainsi de la Terre sainte jordanienne chrétienne, également revendiquée par la monarchie mais à des fins touristiques plutôt et liée à la promotion du rapprochement islamo-chrétien. Elle s'oppose en tout cas à la Terre sainte juive, correspondant au territoire d'Israël de l'autre côté de la frontière. Des textes du ministère des waqf-s glorifient les Compagnons du Prophète pour leurs qualités exceptionnelles et leur bravoure. Ils font particulièrement référence à deux compagnons : Ja'far b. Abi Talib et Abu 'Ubayda b. al-Jarrah. Concernant le premier, le site Internet du ministère des waqf-s rapporte ces faits :

«Au cours de la bataille de Mu'ta, on le vit trouver la mort, tenant la bannière de l'islam de sa main droite, puis après qu'elle eût été coupée, de sa main gauche, puis après que celle-ci ait été coupée, il serra la bannière avec le haut de ses bras et s'adressa aux musulmans en scandant : comme le Paradis est proche!" (ministère des waqf-s, 2006).

Cependant, la figure de ce Compagnon permet surtout de construire une continuité de la présence hachémite sur le territoire jordanien. Le père de Ja'far, Abu Talib, était le frère du père du Prophète ; il semble avoir été le chef de clan des Hashim et protégea son neveu contre les persécutions des Mecquois (Watt, 1960). Selon The Holy Sites in Jordan (Bin Muhammad, 1999 : 56), Ja'far était la personnalité qui ressemblait le plus "physiquement et moralement " au Prophète. De son côté, Abu 'Ubayda b. al-Jarrah est présenté comme le amìn al-umma, "le gardien de la communauté " (ministère des waqf-s, op. cit.), et symbolise dans la construction discursive la piété et la fidélité. Il appartenait au 
cercle des conseillers et des proches du Prophète (Gibb, 1995). La reconstruction de son mausolée permet ainsi de mettre en valeur une fois de plus l'importance du territoire jordanien pour le Prophète lui-même, mais elle permet surtout de rapprocher l'histoire des Compagnons de celle de la famille régnante.

Les Compagnons sont assimilés à la famille royale par le biais de l'appartenance commune à l'Abl al-bayt, ou du moins au cercle des personnalités très proches du Prophète. Ainsi, l'évocation récurrente de certains d'entre eux permet à la monarchie hachémite de réécrire l'histoire islamique de la Jordanie. Cette réécriture passe par la création d'une continuité entre l'histoire des premiers temps de la conquête du Bilad al-Sham et l'histoire de la Jordanie contemporaine, mais aussi entre les héros de la conquête et les monarques actuels. Ainsi, le processus de sacralisation de la Jordanie est axé sur plusieurs objectifs : offrir au pays de nouveaux lieux de mémoire en évoquant un passé glorieux et présenter une continuité historique entre les premiers temps de l'islam et la Jordanie contemporaine. Ce processus donne alors de "la profondeur historique " à la Jordanie et ses monarques.

\section{Enjeux archéologiques et touristiques}

La gestion faite par le ministère des waqf-s de ces mausolées n'est pas sans rappeler les problématiques plus générales liées au patrimoine en Jordanie. Rappelons que les lieux saints musulmans sont gérés par le ministère des waqf-s alors que les lieux saints chrétiens le sont par le ministère du Tourisme et des Antiquités. Comme nous l'avons évoqué, l'architecture des mausolées musulmans, ici mamelouks et ottomans, n'a pas été l'objet d'une volonté de conservation. Les travaux de Rami Daher (2007) et d'Irene Maffi (2004) démontrent que le concept de patrimoine ne s'applique pas, en Jordanie, aux sites liés à l'histoire moderne et contemporaine. Ce patrimoine est marginalisé dans l'histoire officielle : une dissociation est opérée entre l'histoire jordanienne et l'histoire de l'Empire ottoman et des traditions locales. Il s'agit de rebâtir une légitimité historique basée sur une civilisation plus distante, celle des Nabatéens. Le mouvement de reconstruction des lieux saints participe de la même volonté d'occulter les patrimoines mamelouk et ottoman.

Cependant, la particularité de ce mouvement est la recréation d'un patrimoine historique islamique jordanien, qui valorise exclusivement les premières conquêtes islamiques et la période omeyyade. Ainsi, au nord de Ajlun, a été reconstruite une mosquée de type omeyyade sur le site de Listib, qui, selon la tradition, correspond au village d'origine du prophète Élie. Selon l'architecte en charge des rénovations, aucune fouille n'a été effectuée et aucun élément ne permet de dater avec certitude le bâtiment. De la même manière, on trouve, au 
sud d'Amman, un site supposé être la grotte des Sept Dormants ${ }^{20}$, sur lequel le Comité royal a construit une mosquée, donnant ainsi un caractère islamique à ce site commun aux traditions chrétienne et musulmane. Pourtant, les panneaux touristiques $\mathrm{du}$ "Museums with no Frontiers » ${ }^{21}$ insistent sur la présence omeyyade en Jordanie et sur les bonnes relations entre chrétiens et musulmans dès cette époque. Le ministère des waqf-s s'appuie principalement sur des références au patrimoine omeyyade au détriment d'un patrimoine plus récent. La rénovation des lieux saints participe donc du mouvement de valorisation du patrimoine islamique omeyyade entamé à la fin des années quatre-vingt-dix (Maffi, op. cit.) Par ailleurs, le choix des Omeyyades s'explique par la volonté de valoriser des peuples considérés comme indigènes, mais aussi par leur proximité avec l'Abl al-bayt, ce qui les rapproche de la dynastie hachémite. On retrouve cette volonté d'affirmer la spécificité strictement islamique de la Terre sainte jordanienne qui est mise en relation avec l'âge d'or de l'islam : la conquête islamique du Bilad al-Sham et le règne de la dynastie omeyyade. C'est à partir de cette définition que le ministère des waqf-s jordanien a tenté de développer une nouvelle activité touristique autour des lieux saints nationaux.

\section{Autres lieux, autres pratiques : vers un tourisme islamique}

Le tourisme est un secteur économique extrêmement sensible aux évolutions de la situation politique et tous les pays du Moyen-Orient ont vu leur fréquentation touristique baisser après les attentats du 11 septembre 2001. À partir de cette date, une coopération s'était instaurée entre les pays arabes et les pays musulmans concernant les questions touristiques. Cette coopération s'est intensifiée et se matérialise par la prolifération de traités et d'accords bilatéraux ou régionaux (Al-Hamarneh, Steiner, 2004). Le développement du tourisme islamique est donc un phénomène récent qui tend à prendre de l'ampleur dans l'ensemble du monde arabe. Ce secteur d'activité est conçu, d'après l'éditeur de la revue Islamic Tourism ${ }^{22}$, Abdel-Sahib al-Shakry, selon trois objectifs : " raviver la culture et les valeurs islamiques, apporter des bénéfices économiques aux sociétés islamiques et renforcer la confiance envers l'islam face aux préjugés des autres religions ".

20. Cette tradition concerne sept jeunes gens de confession chrétienne qui auraient fui les persécutions de l'empereur Dèce au III ${ }^{e}$ siècle et se seraient réfugiés dans une cave dans laquelle ils s'endormirent pendant trois siècles. Ils se réveillèrent sous le règne de l'empereur Théodore, alors que l'ensemble de l'Empire était converti au christianisme. Cette tradition est commune à l'islam (sourate XVIII, 8-15, "La Caverne ») et au christianisme (Sourdel, 2004).

21. Ce programme, financé par l'Union Européenne depuis la fin des années quatre-vingtdix, est entièrement dédié à l'héritage omeyyade.

22. La revue Islamic Tourism, créée en 2004, est publiée en plusieurs langues et a pour fonction de valoriser les différents sites ou villes islamiques. Elle cherche à toucher un public musulman large et varié. 
La Jordanie a très tôt voulu participer à cette industrie dont le développement est pourtant lent dans le pays. La reconstruction des mausolées nationaux fut dans les premiers temps envisagée comme un moyen pour les populations locales de réinvestir ces sites. L'architecte Rasem Badran ${ }^{23}$ explique que le marché aménagé autour du mausolée d'Abu 'Ubayda b. al-Jarrah était destiné aux populations locales. À partir des années deux mille, la fonction de ce marché a totalement changé. L'objectif du ministère des waqf-s est aujourd'hui d'en faire un marché touristique :

" [les activités du marché] seront définies de manière à ne pas entrer en contradiction avec l'importance spirituelle et religieuse du site et se limiteront aux éléments suivants : une bibliothèque, des marchands de souvenirs et d'artisanat, des exemplaires du Coran et des rosaires, un office de tourisme, (...) des boutiques de cadeaux » (Comité royal, $2000: 23$ ).

La plupart des panneaux de signalisation du site sont traduits en plusieurs langues dont le persan et l'ourdou, indiquant la volonté de voir sa fréquentation augmenter et se diversifier. De la même manière, le dernier projet de reconstruction du site de Mu'ta évoqué plus haut prévoit de mettre à la disposition des visiteurs des chevaux permettant de se rendre des mausolées au site de la bataille situé à trois kilomètres dans la ville de Mu’ta. Depuis 2001, ces mausolées, conçus à l'usage des populations locales, sont investis d'une nouvelle fonction touristique.

Le programme de développement du tourisme islamique a été élaboré en collaboration avec les experts de l'USAID qui ont désigné, dans le plan de développement pour 2004-2010, le tourisme religieux comme l'un des secteurs-clés pour intensifier l'industrie touristique. Le mouvement de reconstruction des lieux saints a donc d'abord été un moyen de contrôler des lieux de sociabilité locaux et d'en redéfinir les usages. À partir de 2001, une fonction de support d'un tourisme islamique a été associée à ces monuments. Le processus ne pouvait opérer que dans un rapport conflictuel aux usages traditionnels de ces édifices et sites. Les sociétés locales ont donc été confrontées à un processus de désappropriation. L'émergence du tourisme chiite a notamment engendré de nouvelles pratiques de piété.

Ce tourisme s'est développé autour du mausolée de Ja'far b. Abi Talib. Pour les chiites, Ja'far b. Abi Talib, frère de l'imam Ali et cousin du Prophète, est considéré comme un saint. Un pèlerinage chiite s’y déroulait, avant 2006, principalement au moment de l' 'ashūra ${ }^{24}$, et il semble que le ministère du Tourisme jordanien ait souhaité développer des accords avec l'Iran pour faciliter la circulation et l'accueil des pèlerins. On trouve ainsi, sur un site d'information jordanien, un article relatif au développement du tourisme chiite en Jordanie :

23. Entretien avec Rasem Badran, architecte, Dar al-Omran, Amman, 14 mai 2009.

24. Fête chiite qui commémore le martyre de Hussein à la bataille de Kerbela. 
«Plus de 3000 pèlerins ont visité les tombes des Compagnons du Prophète Muhammad et des martyrs de la bataille de Mu'ta dans la première moitié de l'année 2005. Le directeur du département des waqf-s de Mazar-sud, Firas Abu Kheit, a dit que des pèlerins iraniens viendraient bientôt visiter ce site conformément aux accords iraniensjordaniens en matière de tourisme religieux " (Hijjawi, 2005).

Le développement du tourisme chiite en Jordanie fut, au cours des années 2002-2006, un objectif du ministère des waqf-s et la fréquentation du mausolée de Ja'far b. Abi Talib a augmenté entre 2004 et 2005. Ce phénomène n'est pas sans rappeler les visites massives aux mausolées à Damas et à Raqqa, en Syrie. Myriam Ababsa, Sabrina Mervin et Paolo Pinto (op. cit.) les expliquent par la fermeture des lieux saints de Najaf et de Kerbela après la guerre Iran-Irak, de 1980 jusqu'en 2003, alors que l'Arabie Saoudite imposait un quota d'entrée pour les pèlerins à la Mecque. C'est seulement depuis 2003, et la chute du régime irakien, que le pèlerinage à Kerbela est de nouveau possible. Par ailleurs, dans le cas jordanien, il convient de rappeler que, depuis la guerre du Golfe, le mausolée a reçu les visites de migrants irakiens réfugiés en Jordanie, ce qui leur permettait de rester en relation avec la culture irakienne. Certains d'entre eux considéraient le mausolée comme une zone de contact avec les pèlerins venus d'Irak-même (Chatelard, 2005 : 3). Cependant, depuis l'exécution de Saddam Hussein en 2006, et le discours du roi Abdallah II, en 2004, concernant la menace d'un axe chiite au Moyen-Orient, l'accès à ce site est étroitement surveillé et limité. Depuis 2006, la constitution d'un espace de sociabilité irakienne ainsi que la présence de pèlerins chiites apparaissent pour les dirigeants jordaniens comme une menace pour la stabilité politique du pays.

Le tourisme islamique reste toutefois un phénomène mineur et marginal en Jordanie. Selon des professionnels ${ }^{25}$ organisant des tours islamiques, ces programmes ont surtout la faveur de touristes d'origine malaise, indonésienne ou turque (tous sunnites). La plupart visitent les lieux saints jordaniens à l'occasion d'un voyage à visée religieuse dans la région. Le tourisme islamique se rattache majoritairement à deux autres types de visites. Tout d'abord une partie des fidèles se rend aux mausolées jordaniens lors du pèlerinage à la Mecque et Médine. De nombreuses agences de voyage proposent des tours incluant la visite des deux villes saintes du Hedjaz puis de Jérusalem. Les pèlerins transitent alors par Amman d'où ils effectuent généralement une visite d'un jour des mausolées, principalement ceux de la vallée du Jourdain. Par ailleurs, une autre partie des touristes effectue cette visite dans le cadre d'une tournée dans le Moyen-Orient, incluant en priorité la visite des lieux saints de Syrie ou de Palestine. On a ainsi assisté, entre les années 1990-2009, à une évolution de la fonction des mausolées, désertés par leurs habitués locaux mais de plus en plus fréquentés par des pèlerins en provenance de pays musulmans limitrophes ou plus lointains. De fait, ces sites sont très peu visités, que ce soit par les touristes ou la population locale.

25. Entretien avec des professionnels du tourisme, Amman, mai-juin 2009. 


\section{Tourisme et représentation : diffusion de l'idéologie nationale}

Dans leur ouvrage consacré au tourisme dans le Tiers-Monde, Martin Mowforth et Ian Munt (2004) démontrent que le tourisme est un puissant facteur de représentation de soi-même à l'extérieur des frontières d'un pays. Dans cette perspective, les lieux saints sont investis d'un rôle représentatif majeur à l'échelle internationale. Par exemple, le site d'Abu 'Ubayda b. al-Jarrah est doté d'un hall VIP destiné à recevoir les hôtes importants : le roi y accueille de nombreux présidents et ministres des waqf-s de pays étrangers. Le tourisme devient ainsi un vecteur de propagation de l'idéologie hachémite. Il est un moyen de diffusion dans le monde musulman de l'image de la Jordanie comme terre sainte islamique et des Hachémites comme représentants privilégiés et porte-parole de la communauté musulmane. Dans ce contexte, le message d'Amman joue un rôle fondamental dans la rhétorique officielle. Il s'adresse aux oulémas du monde entier dont il définit les responsabilités, précisant :

"C'est en les oulémas de notre umma que nous plaçons l'espoir qu'ils mettront tout en œuvre pour éclairer l'esprit de nos jeunes générations sur la réalité de l'islam et ses valeurs sublimes, de sorte à leur éviter de verser dans les aléas de l'ignorance, de la corruption, de l'introversion et de la dépendance. Nous comptons sur eux pour qu'ils leur inculquent les principes de la tolérance, de la modération et du bien, tout en les éloignant des dangers de l'extrémisme. " (Message d'Amman, op. cit., note 15, trad. de l'auteure).

Ce message peut être interprété comme un moyen pour les Hachémites de se présenter comme les garants d'un islam modéré et, de ce fait, comme les interlocuteurs idéaux au Moyen-Orient dans les milieux diplomatiques. De la même manière, la monarchie hachémite se présente, depuis les années quatre-vingt, comme le porte-parole musulman du dialogue islamo-chrétien, principalement par l'intermédiaire du prince Hassan, qui a créé, en 1994, le Royal Institute for Interfaith Studies.

\section{Conclusion}

La Jordanie représente un cas original de création massive de lieux saints, principalement à partir de lieux de culte préexistants. À partir des années quatrevingt-dix et à l'initiative de la monarchie, une grande partie des lieux saints a été reconstruite, dans des styles architecturaux et artistiques nouveaux qui marquent profondément le paysage jordanien et redessinent tant une topographie qu'une hagiographie musulmane sainte et officielle. Cette entreprise correspond à la volonté hachémite d'inclure la Jordanie à la Terre sainte musulmane. Cette nouvelle définition du territoire national s'appuie sur l'affirmation de sa spécificité en tant que terre de l'Abl al bayt tout d'abord, alors que la Palestine est la " terre des prophètes", puis en tant que terre chérie par le Prophète lui-même. La monarchie essaie alors de créer un particularisme d'un autre type en inventant une continuité entre l'histoire de la conquête islamique du Bilad al-Sham et celle 
de son territoire, mais aussi entre les Compagnons du Prophète et la famille hachémite. Cette dernière apparaît ainsi comme la garante privilégiée de la Terre sainte musulmane.

De lieux de mémoire, les sites rénovés sont devenus aux yeux de la monarchie des lieux représentatifs du patrimoine historique de l'islam. Alors qu'ils étaient initialement investis par les populations locales, ils tendent aujourd'hui à accueillir des touristes musulmans venus du monde entier. Cette activité touristique, qui pourrait permettre le développement de ces zones économiquement défavorisées du Royaume, reste cependant marginale. Elle est considérée par la monarchie comme un moyen de véhiculer dans l'ensemble du monde musulman une image des Hachémites comme porte-parole de la communauté musulmane et garants du message prophétique. De cette manière, la monarchie cherche à donner à son pays un rôle privilégié au sein du monde arabe, en particulier par rapport à l'Arabie Saoudite. Cette volonté de représentation se manifeste notamment par l'important engagement de la monarchie dans les conférences internationales relatives aux dialogues inter- et intra-religieux. La création d'une topologie sacrée en Jordanie vise ainsi deux objectifs majeurs : la réécriture de l'histoire islamique nationale et la définition de la Jordanie comme Terre sainte musulmane, mais aussi la redéfinition de la fonction même de ces lieux de piété. Cependant, la fréquentation limitée de ces sites pose la question de l'efficacité de ce mouvement de reconstruction.

Norig NEVEU

CRH-EHESS

norigneveu@hotmail.fr

\section{Bibliographie}

AbabSA Myriam, 2001, "Les mausolées invisibles : Raqqa, ville de pèlerinage chiite ou pôle étatique en Jazîra syrienne », Annales de géographie, 622, pp. 647-664.

Abel A., 1960, "Bahîrâ ", in Encyclopédie de l'islam, NE-I, Leiden, Paris, E. J. Brill, Maisonneuve et Larose, pp. 950-951.

AL-'Adwa Samîr, AL-ZANî̉ÂT Muhammad, 2005, « Maqāmāt al-sahāba wa 'adrha alšuhadā' : fadā' 'ābiq bil-mukaramāt iarūtu 'ahfād al-hāšimiȳn bi-'ajdādhum » [Les mausolées de Compagnons du Prophète et les tombeaux de martyrs : des espaces bénis reliant les descendants hachémites à leurs grands-pères], al-Ghad, http://www.alghad. com/, 24 avril 2005.

al-Hamarneh Ala, Steiner Christian, 2004, « Islamic Tourism: Rethinking the Strategies of Tourism Development in the Arab World after September 11, 2001 », Comparative Studies of South Asia, Africa and the Middle East, 24-1, pp. 73-182.

Aubin-Boltanski Emma, 2004, Prophètes héros et ancêtres. Les pèlerinages musulmans de Nab̂̂ Mûsâ et Nabî Salîh dans la construction nationale palestinienne, thèse de doctorat en anthropologie, Paris, EHESS. 
Bauman Joseph, 1995, "Designer Heritage. Israeli National Parks and the Politics of Historical Representation ", Middle East Report, sept.-oct., pp. 16-19.

Benvenisti Meron, 2000, Sacred Landscape. The Buried History of the Holy Land since 1948, Berkeley, Los Angeles, University of California Press.

Bin Munammad Ghazi (Prince), (ed.), 1999, The Holy Sites of Jordan, Amman, Turab.

Chatelard Géraldine, 2004, Briser la mosaïque. Lien social et identités collectives chez les chrétiens de Madaba, Jordanie (1870-1997), Paris, CNRS Éditions.

-, 2005, «Un système en reconfiguration : l'émigration des Irakiens de la guerre du Golfe à la guerre d'Irak (1990-2003) ", in Jaber H., Métral F., (éds.), Monde en mouvement au Moyen-Orient au tournant $d u \mathrm{XX}^{e}$ siècle, Beyrouth, Institut Français du ProcheOrient, pp. 113-155.

Comité royal pour la construction de mosquées et de mausolées de prophète, de Compagnons et de martyrs, Hashemite Construction of the Grand Islamic Complex where Mu'ta Commanders are Buried, Amman, $24 \mathrm{p}$.

DAHER Rami, 2007, « Tourism, Heritage and Urban Transformation in Jordan and Lebanon: Emerging Actors and Global-Local Juxtaposition ", in Daher R., (ed.), Tourism in the Middle East, Continuity, Change and Transformation, Clevedon, Channel View Publications, pp. 263-307.

Dawn C. E., 1971, "Hashimides ", in Encyclopédie de l'islam, NE-III, Leiden, Paris, E. J. Brill, Maisonneuve et Larose, pp. 270-272.

Giвв H. A. R., 1995, "Abû 'Ubayda b. Al-Djarrâh », in Encyclopédie de l'islam, NE-I, Leiden, E. J. Brill, Paris, Maisonneuve et Larose, p. 163.

Halbwachs Maurice, 1971, La topographie légendaire des évangiles en Terre Sainte, Paris, Presses Universitaires de France.

HiJjAw Ahmad, 2005, «3,000 pilgrims visit Muta and Mazar », at www.jordan.jo, 19 sept. 2005.

Jordan Tourism Board, 2002, Tracing Islam in Jordan, Amman, 20 p.

Katz Kimberly, 2001, Holy Places and National Spaces: Jerusalem under Jordanian Rule, thèse de doctorat, New York, New York University.

-, 2003, « Legitimizing Jordan as the Holy Land: Papal Pilgrimages, 1964-2000 », Comparative Studies of South Asia, Africa and the Middle East, 23/1-2, pp. 181-189.

LEWICKI Tadeusz, 1971, " al-Ibâdiyya ", in Encyclopédie de l'islam, NE-III, Leiden, Paris, E. J. Brill, Maisonneuve et Larose, pp. 669- 682.

MAFFI Irène, 2004, Pratique du patrimoine et politique de la mémoire en Jordanie. Entre histoire dynastique et récits communautaires, Lausanne, Payot.

Mervin Sabrina, 1996, "Sayyida Zaynab, banlieue de Damas ou nouvelle ville sainte chiite ", Cemoti, 22, pp. 149-162.

Ministère des waqf-s et des Affaires islamiques et le département du tourisme et des anti-

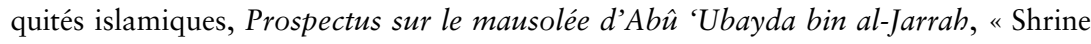
of the Trustee of the Nation Abû 'Ubayda bin al-Jarrah ", s.l.n.d.

Ministère des waqf-s et des Affaires islamiques, 2000, Hashemite Construction of Mosques and Shrines of Prophets and their Companions, and the Most Important Islamic and Historical Sites, Amman, 50 p. 
Ministère des waqf-s et des Affaires islamiques, 2006, " Mašrū' masjid al-sahāba al-Jalāl Ja'far bin Abi Tālib » [Projet pour la mosquée du Compagnon du Prophète Ja'far bin Abî Tâlib], www.awqaf.gov.jo/

Mowforth Martin, Munt Ian, 1998, Tourism and Sustainability. New Tourism in the Third World, Londres, Routledge.

Nora Pierre, 1984, "Entre mémoire et histoire. La problématique des lieux », in Nora P., Les lieux de mémoire, t. 1, Paris, Gallimard, pp. 15-42.

OHANESSIAN-ChARPIN Anna, 1995, " Greniers, maladies, morts, systèmes de stockage, système de valeur chez les bédouins du Ghor ", in Bazzana A., Delaigue M.-C., Ethnoarchéologie méditerranéenne, Madrid, Casa de Velasquez.

PINTo Paolo, 2007, «Pilgrimage, Commodities, and Religious Objectification: The Making of Transnational Shiism between Iran and Syria », Comparative Studies of South Asia, Africa and the Middle East, 27, pp. 109-124.

Prevost Virginie, 2009, «Les mosquées ibadites du Maghreb », in Adelkah F., Moussaoui A., (éds.), «Les mosquées. Espace, institutions et pratiques », REMMM, 125, pp. 217-232.

Rogan Eugène Lawrence, 1986, "Physical Islamization in Amman », The Muslim World, 426-427/1, pp. 24-42.

Sourdel Janine, Sourdel Dominique, 2004, « Hachimides ou Banû Hâshim », in Sourdel J., Sourdel D., (éds.), Dictionnaire historique de l'islam, Paris, Presses Universitaires de France, pp. 323-324.

-, 2004, "Caverne (gens de la) ", in Sourdel J., Sourdel D., Dictionnaire historique de l'islam, Paris, Presses Universitaires de France, p. 188.

Veccia Vaglesi L., 1965, "Dja'far bin Abî Tâlib », in Encyclopédie de l'islam, NE-II, Leiden, Paris, E. J. Brill, Maisonneuve et Larose, p. 382.

WALKER Bethany, 2007, "Interpreting Welfare and Relief in the Middle East ", in Naguib N., Okkenhaug I. M., (eds.), Social, Economic and Political Studies of the Middle East and Asia, 103, pp. 217-234.

WATT Montgomery, 1960, "Abû Talib », in Encyclopédie de l'islam, NE-I, Leiden, Paris, E. J. Brill, Maisonneuve et Larose, p. 157.

\section{Résumé}

Depuis les années quatre-vingt-dix, le roi Hussein puis son fils, le roi Abdallah II ont entrepris la reconstruction des mausolées (maqām) de prophètes antéislamiques et Compagnons du Prophète Muhammad sur l'ensemble du territoire jordanien, en particulier le long de la vallée du Jourdain. La reconstruction de ces sites révèle une volonté d'innovation architecturale et artistique et a engendré la disparition de formes architecturales anciennes. Cette entreprise a permis à la monarchie une réécriture de l'histoire de l'islam en Jordanie dans le but de créer une épaisseur historique entre l'âge d'or de la conquête islamique du Bilad al-Sham et la Jordanie contemporaine. La monarchie jordanienne cherche ainsi à faire de ces mausolées des lieux de mémoire jordaniens. Cet article cherchera à comprendre les différentes étapes de la sacralisation du territoire jordanien et de son assimilation à la Terre sainte musulmane. Nous tenterons ainsi d'analyser les enjeux politiques et économiques de cette redéfinition $d u$ territoire national.

Mots-clés : sacralisation, lieux de mémoire, Terre sainte, tourisme, Jordanie. 


\section{Abstract}

Since the 1990s, Late King Hussein and his son, King Abdullah II, rebuilt mausoleums (maqām) of pre-Islamic Prophets and Companions of Prophet Muhammad all over the Jordan national territory, especially along the Jordan Valley. Rebuilding these sites reveals a drive of innovation at architectural and artistic levels but also leads to the disappearance of former architectural forms. It was an opportunity for the Monarchy to rewrite the Islamic history of Jordan and to provide the country with historical continuity between the first time of the Islamic conquest of Bilad alSham and contemporary Jordan. These holy sites tend to be used as memorial sites for the Monarchy. This article will try to understand the different steps of the sacralization of the Jordanian national territory seeking to include it in the Islamic Holy Land. It will try to analyze the political and economic strategies around this redefinition of the national territory.

Key words: sacralization, memorial sites, Holy Land, tourism, Jordan.

\section{Resumen}

Desde la década de 1990, el rey Hussein y su hijo, el rey Abdallab II han emprendido la reconstrucción de los mausoleos (maqām) de los profetas y de los Compañeros del Profeta Muhammad sobre todo el territorio de Jordania y en particular a lo largo del valle del Jordán. La reconstrucción de estos lugares revela un deseo de innovación arquitectónica y artística, pero también ha provocado la desaparición de la forma arquitectónica anterior. La renovación de los mausoleos supuso para la monarquía una ocasión de reescribir la historia del Islam en Jordania con el fin de reinventar une continuidad histórica entre los primeros tiempos de la conquista islámica del Bilad al-Sham y la Jordania contemporanea. La monarquía jordana aspira a hacer de estos santuarios lugares de memoria de Jordania. Este artículo intenta entender las diferentes etapas de la sacralización del territorio nacional y su asimilación en la Tierra Santa islámica. Tratamos de entender las estrategias políticas y económicas de esta redefinición del territorio nacional.

Palabras clave: sacralización, lugar de memoria, Tierra Santa, turismo, Jordania. 